\title{
Acidic Dissociation Constant and Related Thermodynamic Quantities for Monoethanolammonium Ion in Water From $0^{\circ}$ to $50^{\circ} \mathrm{C}$
}

\author{
Roger G. Bates and Gladys D. Pinching
}

\begin{abstract}
Twenty-three buffer solutions composed of approximately equal molal amounts of monoethanolamine (2-aminoethanol) and monoethanolammonium chloride in water were studied by electromotive-force methods at 11 temperatures from $0^{\circ}$ to $50^{\circ} \mathrm{C}$. The values obtained for the acidic dissociation constant, $K_{b h}$, of the ethanolammonium ion are given in this range of temperatures by

$$
-\log K_{b h}=2677.91 / T+0.3869+0.0004277 T,
$$

where $T$ is the temperature on the Kelvin scale. The changes of free energy, heat content, entropy, and heat capacity that accompany the dissociation of 1 mole of ethanolammonium ion in the standard state were calculated from the dissociation constant and its temperature coefficient. Dissociation of a mole of ethanolammonium ion results in a small decrease of heat capacity. In this respect ethanolammonium ion resembles ammonium ion rather than the methyl-substituted ammonium ions, for which rather large increases of heat capacity on dissociation have been found.
\end{abstract}

\section{Introduction}

During the past 20 years much attention has been given to the effect of temperature changes on the dissociation constants of neutral and negatively charged acids. These studies have given a precise knowledge of the thermodynamic quantities associated with the dissociation of acids of these types. Nevertheless, the strengths of only a few positively charged acids have been measured both with the precision attainable by modern techniques and over a sufficiently wide range of temperatures to warrant the computation of the changes of entropy, heat content, and heat capacity that accompany the dissociation process.

The dissociation of a monobasic cationic acid is an isoelectric reaction; a proton is shifted, but no new electrostatic charge is created. The electrostatic contribution to the change of heat capacity, $\Delta C_{p}^{\circ}$, should therefore be zero, and it has been predicted that $\Delta C_{p}^{\circ}$ for these dissociative processes would itself be found to be small or zero $[1,2] .^{1}$ Although this prediction was confirmed for ammonium ion [3], later studies of the methylammonium ions gave values of $\Delta C_{p}^{\circ}$ ranging from $+33 \mathrm{j} \mathrm{deg}^{-1}$ mole $^{-1}$ for monomethylammonium to $+183 \mathrm{j}$ $\mathrm{deg}^{-1}$ mole $^{-1}$ for trimethylammonium [4]. This discovery led Everett and Wynne-Jones to the conclusion [4] that specific interactions of a chemical nature among the solvent and the dissolved ions and molecules often exert a more profound influence upon the heat capacity than expected.

Everett and Wynne-Jones attributed the positive values of $\Delta C_{p}^{\circ}$ for the substituted ammonium ions to the strongly hydrophobic character of the alkyl groups. This hydrophobic property is reduced by substitution of hydroxyl on the alkyl group. Hence, it is of

1 Figures in brackets indicate the literature references at the end of this paper. interest to compare the thermodynamic functions for the dissociation of ethanolammonium ion with those for ammonium and the methyl-substituted ammonium ions. The dissociation constant of monoethanolammonium ion and the basic strength of monoethanolamine were determined by the electromotive-force method from $0^{\circ}$ to $50^{\circ} \mathrm{C}$ and the thermodynamic quantities for the dissociation processes calculated. The change of heat capacity for the dissociation of monoethanolammonium ion in the standard state at $25^{\circ}$ was found to be $-5 \mathrm{j}$ $\mathrm{deg}^{-1}$ mole $^{-1}$, not greatly different from that for ammonium ion.

\section{Method}

The method used was essentially the same as that by which the dissociation constant of ammonium ion was determined [5]. Electromotive-force measurements of the cell

$$
\begin{gathered}
\mathrm{Pt} ; \mathrm{H}_{2}(g, 1 \mathrm{~atm}), \mathrm{HOC}_{2} \mathrm{H}_{4} \mathrm{NH}_{3} \mathrm{Cl}\left(m_{1}\right), \\
\mathrm{HOC}_{2} \mathrm{H}_{4} \mathrm{NH}_{2}\left(m_{2}\right), \mathrm{AgCl}(s) ; \mathrm{Ag},
\end{gathered}
$$

were made at intervals of $5 \mathrm{deg}$ from $0^{\circ}$ to $50^{\circ}$. The partial pressure of the amine from its aqueous solution was so low that no correction for volatility of the solute was necessary, and the extra saturator used in determining the dissociation constant of ammonium ion was not required. Furthermore, it was found that the correction for solubility of silver chloride in these buffer solutions is only about 0.0006 in $\log K_{b h}(0.00004 \mathrm{v}$ in the emf) and can therefore be neglected.

The acidity function, $\mathrm{pwH}$, was computed for each buffer solution at each temperature from the emf, $E$, the standard potential of the cell, $E^{\circ}[5]$, 
and the appropriate value of $2.3026 R T / \boldsymbol{F}$ [5] by the equation

$$
\begin{gathered}
\mathrm{pwH} \equiv-\log \left(f_{\mathrm{H}} f_{\mathrm{Cl}} m_{\mathrm{H}}\right)=\left(E-E^{\circ}\right) \boldsymbol{F} /(2.3026 R T)+ \\
\log m_{\mathrm{Cl}},
\end{gathered}
$$

where $m$ is molality and $f$ is the molal activity coefficient. If the mass-law expression for the dissociation of ethanolammonium ion, $\left(\mathrm{BH}^{+}\right)$, into ethanolamine (B) and hydrogen ion, namely,

$$
\mathrm{BH}^{+}=\mathrm{B}+\mathrm{H}^{+}
$$

is combined with eq 1 , the following relationship between the dissociation constant for eq 2, namely $K_{b h}$, and pwH is obtained:

$$
\begin{aligned}
-\log K_{b h}= & \mathrm{pwH}+\log \left(m_{\mathrm{BH}^{+}}+m_{\mathrm{B}}\right)+ \\
& \log \left(f_{\mathrm{BH}^{+}} f_{\mathrm{Cl}^{-}} / f_{\mathrm{B}}\right) .
\end{aligned}
$$

\section{The Molality Term}

Inasmuch as the basic strength of ethanolamine is greater than the acidic strength of ethanolammonium ion, its conjugate acid, the solutions have an alkaline reaction. The basic dissociation is formulated as follows:

$$
\mathrm{B}+\mathrm{H}_{2} \mathrm{O}=\mathrm{BH}^{+}+\mathrm{OH}^{-} ;
$$

and the basic dissociation constant is designated $K_{b}$. The concentration of hydroxyl ion evidently measures the amount of free amine that has been converted by reaction 4 into the ion $\mathrm{BH}^{+}$. Hence, the equilibrium concentrations of $\mathrm{B}$ and $\mathrm{BH}^{+}$are

and

$$
m_{\mathrm{BH}^{+}}=m_{1}+m_{\mathrm{OH}^{-}},
$$

$$
m_{\mathrm{B}}=m_{2}-m_{\mathrm{OH}^{-}}
$$

and the ionic strength, $\mu$, is given by

$$
\mu=m_{1}+m_{\mathrm{OH}^{-}} .
$$

The concentration of hydroxyl ion is easily computed by the approximation

$$
\log m_{\mathrm{OH}^{-}} \approx \log K_{w}+\mathrm{pwH},
$$

where $K_{w}$ is the ionization constant of water [6].

\section{The Activity-Coefficient Term}

The last term of eq 3 approaches zero at high dilutions. However, it is not feasible to omit this term and simply to extrapolate the sum of the first two terms on the right to zero ionic strength, for the last term contributes nearly 0.1 to $\log K_{b h}$ at an ionic strength of 0.01 , and accurate data are often unobtainable at ionic strengths below 0.01. The Hückel equation [7] offers a practical means of estimating the activity-coefficient correction. The complete expression for $K_{b h}$ is obtained by combining eq 3 with the Hückel equation and with eq $5 \mathrm{a}$ and $5 \mathrm{~b}$ :

$$
\begin{array}{r}
-\log K_{b h}^{\prime} \equiv-\log K_{b h}-\beta \mu=\mathrm{pwH}+ \\
\log \frac{m_{1}+m_{\mathrm{OH}}}{m_{2}-m_{\mathrm{OH}}}-\frac{2 A \sqrt{\mu}}{1+B a^{*} \sqrt{\mu}}
\end{array}
$$

In eq $8, K_{b h}^{\prime}$ is the apparent value of $K_{b h}$, the thermodynamic dissociation constant; $A$ and $B$ are constants of the Debye-Hückel theory [8]; and $a^{*}$ and $\beta$ are adjustable parameters. When too large a value of the ion-size parameter, $a^{*}$, is used, a plot of the right side of eq 8 as a function of ionic strength is concave downward. For too small values of $a^{*}$, the curve is concave upward. At each temperature, the intermediate value of 1.0 furnished straight lines, which were easily extended to an ionic strength of zero. The data for five temperatures are plotted in figure 1 .

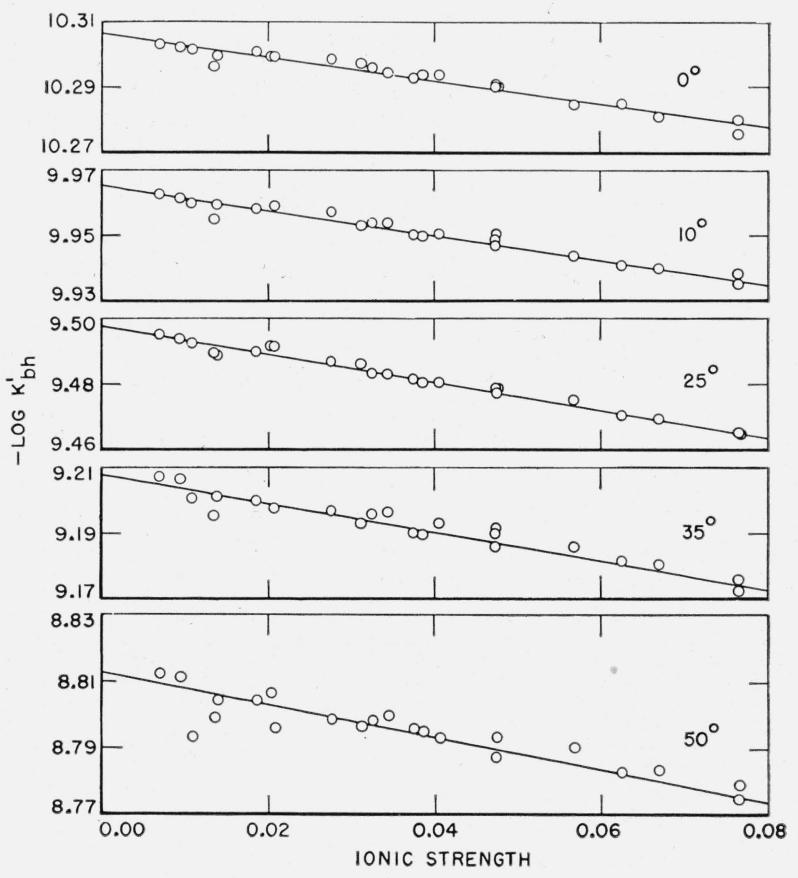

FIGURE 1. Extrapolation of - $\log K_{b h}^{\prime}$, the right side of eq 8 , to zero ionic strength at $0^{\circ}, 10^{\circ}, 25^{\circ}, 35^{\circ}$, and $50^{\circ}$.

\section{Experimental Procedures and Results}

Monoethanolamine (2-aminoethanol) was distilled twice at atmospheric pressure and the middle third of the distillate collected. Two stock solutions of the amine in carbon dioxide-free water were used in preparing the 23 buffer solutions. These two solutions were stored in paraffin-lined flasks and were protected from contamination by atmospheric carbon dioxide and guarded from strong light. Each stock solution was standardized by weight titration with a solution of distilled hydrochloric acid which, in turn, had been standardized by weighing the chloride as silver chloride. The sodium salt of methyl red was chosen as indicator. 
The buffer solutions were grouped into five series. The most concentrated solution of each series was prepared by mixing accurately weighed portions of amine solution and hydrochloric acid solution. Amine and acid were combined in the ratio of 2 moles to 1 . The other solutions of each series were formed by weight dilution of the most concentrated solution with carbon dioxide-free water.

The two electrode compartments of the cells were separated by a stopcock of large bore [5], and air was excluded from the cells during the operations of rinsing and filling. The preparation of the hydrogen and silver-silver chloride electrodes has been described [9].

The solubility of silver chloride in a $0.1-M$ solution of the amine was determined in the following manner. Freshly precipitated silver chloride was digested overnight at about $90^{\circ}$, washed five times with water, and filtered. An excess of the product was added to $200 \mathrm{ml}$ of the amine solution and the mixture shaken vigorously several times over a period of about $18 \mathrm{hr}$. A clear sample of the supernatant solution was withdrawn, acidified with nitric acid, and the precipitated silver chloride was collected, dried, and weighed. One liter of a $0.1-M$ solution of the amine was found to dissolve 0.0021 mole of silver chloride, or about one-third as much as a $0.1-M$ solution of ammonia. Hence, the correction for solubility could safely be omitted.

The values of pwH listed in table 1 were calculated by eq 1 from the measured emf and the chloride molality $\left(m_{1}\right)$. With $a^{*}=1$ and $m_{\mathrm{OH}}$ obtained by eq $7,-\log K_{b h}^{\prime}$ was derived by eq 8 from each experimental measurement and plotted with respect to ionic strength as shown in figure 1 . The slopes, $-\beta$, of these straight lines were measured at each temperature and used to compute $-\log K_{b h}$ for each solution (eq 8). The averages of these 22 or 23 values are summarized in the second column of table 2, together with the mean deviation from the average value at each temperature. The third column gives $K_{b h}$, whereas the last two columns list the negative logarithm of the basic dissociation constant of ethanolamine, $-\log K_{b}$ (compare eq 4), and $K_{b}$, respectively. The basic constant was obtained from $K_{b h}$ and the ionization constant of water [6] by the equation

$$
K_{b}=K_{w} / K_{b h} .
$$

TABLE 1. $\mathrm{pwH}$ for aqueous mixtures of ethanolammonium chloride $\left(m_{1}\right)$ and ethanolamine $\left(m_{2}\right)$ from $0^{\circ}$ to $50^{\circ}$

\begin{tabular}{|c|c|c|c|c|c|c|c|c|c|c|c|c|}
\hline \multirow{2}{*}{$m_{1}$} & \multirow{2}{*}{$m_{2}$} & \multicolumn{11}{|c|}{ pwH at- } \\
\hline & & $0^{\circ}$ & $5^{\circ}$ & $10^{\circ}$ & $15^{\circ}$ & $20^{\circ}$ & $25^{\circ}$ & $30^{\circ}$ & $35^{\circ}$ & $40^{\circ}$ & $45^{\circ}$ & $50^{\circ}$ \\
\hline 0.07648 & 0.07533 & 10.5168 & 10.3459 & 10.1802 & 10.0195 & 9.8645 & 9. 7160 & 9.5699 & 9.4315 & 9. 2960 & 9.1670 & 9. 0413 \\
\hline .07646 & .07551 & 10.5222 & 10.3496 & 10.1817 & 10.0203 & 9. 8627 & 9. 7173 & 9. 5685 & 9. 4290 & 9. 2938 & 9.1644 & 9.0382 \\
\hline .06699 & .06599 & 10.5072 & 10.3360 & 10.1698 & 10.0093 & 9.8540 & 9. 7051 & 9.5599 & 9.4202 & 9. 2849 & 9.1554 & 9. 0297 \\
\hline .06263 & .06185 & 10.5053 & 10.3332 & 10.1646 & 10.0030 & 9.8471 & 9. 6999 & 9. 5541 & 9. 4149 & 9.2793 & 9.1491 & 9. 0226 \\
\hline .05690 & .05605 & 10.4940 & 10.3222 & 10.1562 & 9.9963 & 9.8414 & 9. 6921 & 9.5472 & 9. 4074 & 9.2729 & 9.1437 & 9.0181 \\
\hline .04746 & .04717 & 10.4880 & 10.3159 & 10.1498 & 9.9886 & 9. 8328 & 9. 6833 & 9. 5376 & 9. 3984 & 9. 2322 & 9.1323 & 9.0058 \\
\hline .04741 & .04732 & 10.4874 & 10.3140 & 10.1469 & 9.9855 & 9. 8292 & 9. 6836 & 9.5360 & 9.3943 & 9.2592 & 9.1301 & 9.0014 \\
\hline .04740 & .04767 & 10.4911 & 10.3184 & 10.1520 & 9. 9920 & 9.8365 & 9. 6872 & 9.5409 & 9. 4017 & 9. 2663 & 9.1373 & 9.0110 \\
\hline .04055 & .04005 & 10.4724 & 10.3001 & 10.1319 & 9.9724 & 9. 8163 & 9. 6663 & 9. 5221 & 9. 3820 & 9. 2457 & 9.1151 & 8. 9876 \\
\hline .03858 & .03858 & 10.4724 & 10.2992 & 10.1310 & 9.9698 & 9.8133 & 9.6660 & 9.5193 & 9. 3784 & 9.2444 & 9.1151 & 8.9890 \\
\hline .03746 & .03700 & 10. 4648 & 10. 2929 & 10.1247 & 9. 9614 & 9. 8029 & 9. 6601 & 9. 5123 & 9. 3720 & 9.2377 & 9.1083 & 8. 9826 \\
\hline .03440 & .03389 & 10.4584 & 10. 2861 & 10.1200 & 9.9595 & 9.8043 & 9.6535 & 9.5099 & 9. 3698 & 9.2340 & 9.1044 & 8. 9782 \\
\hline .03253 & .03232 & 10.4590 & 10.2861 & 10.1193 & 9. 9578 & 9.8001 & 9. 6525 & 9.5074 & 9. 3681 & 9.2322 & 9.1031 & 8. 9758 \\
\hline .03116 & .03110 & 10.4586 & 10. 2857 & 10.1170 & 9.9559 & 9. 7996 & 9.6541 & 9.5036 & 9. 3638 & 9.2279 & 9.1002 & 8. 9717 \\
\hline .02765 & .02780 & 10.4539 & 10.2817 & 10.1150 & 9.9546 & 9.7989 & 9.6482 & 9.5008 & 9. 3609 & 9.2243 & 9.0947 & 8.9673 \\
\hline .02077 & .02073 & 10.4316 & 10. 2613 & 10.0936 & 9. 9323 & 9. 7770 & 9.6293 & 9.4802 & 9. 3377 & 9.2006 & 9.0668 & 8. 9398 \\
\hline .02032 & .02002 & 10.4249 & 10.2530 & & & & 9. 6219 & & & 9.1952 & 9.0648 & 8. 9387 \\
\hline .018619 & .018725 & 10.4296 & 10. 2562 & 10.0889 & 9.9291 & 9.7736 & 9.6232 & 9.4751 & 9. 3356 & 9.1990 & 9.0704 & 8.9435 \\
\hline . 013871 & .013700 & 10.4034 & 10. 2310 & 10.0646 & 9. 9028 & 9.7461 & 9. 5964 & 9.4493 & 9. 3104 & 9.1736 & 9.0427 & 8. 9168 \\
\hline .013494 & .013469 & 10.4033 & 10. 2304 & 10.0635 & 9.9019 & 9.7463 & 9.6009 & 9.4491 & 9. 3085 & 9.1744 & 9.0444 & 8.9155 \\
\hline .010944 & .010877 & 10.3956 & 10.2230 & 10.0557 & 9.8925 & 9. 7342 & 9. 5900 & 9. 4390 & 9. 2997 & 9.1627 & 9. 0330 & 8. 9054 \\
\hline .009510 & .009565 & 10.3946 & 10.2220 & 10.0549 & 9.8945 & 9.7384 & 9. 5884 & 9.4426 & 9. 3031 & 9.1672 & 9.0372 & 8.9105 \\
\hline .007042 & .006998 & 10.3768 & 10.2043 & 10.0373 & 9.8759 & 9.7175 & 9.5710 & 9.4239 & 9.2843 & 9.1477 & 9.0185 & 8.8913 \\
\hline
\end{tabular}

TABLE 2. Summary of the dissociation constants of ethanolammonium ion and ethanolamine from $0^{\circ}$ to $50^{\circ}$

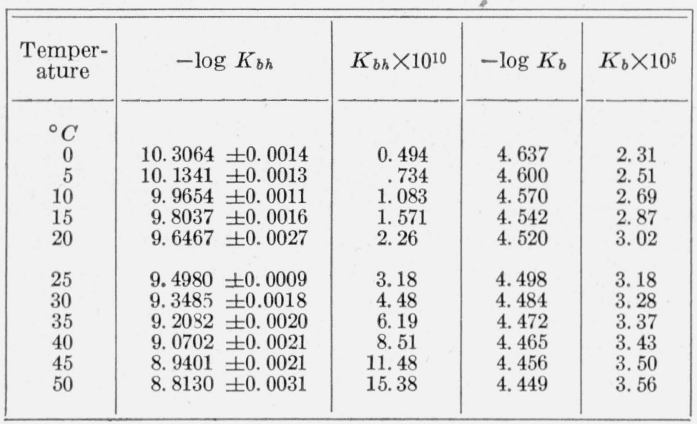

The dissociation constants given in the second column of table 2 may be expressed by the following equation, valid from $0^{\circ}$ to $50^{\circ}$ :

$$
-\log K_{b h}=2677.91 / T+0.3869+0.0004277 T \text {, }
$$

where $T$ is the temperature on the absolute (Kelvin) scale: $T={ }^{\circ} \mathrm{C}+273.16$. The constants of eq 10 were determined by the method of least squares. The average difference at the 11 temperatures between the observed $\log K_{b h}$ and the calculated value was 0.0010 .

Simms [10] found 9.470 for $-\log K_{b h}$ at $25^{\circ}$ from the emf of cells with hydrogen and calomel electrodes, 
whereas Glasstone and Schram [11] obtained 9.45 from measurements made with the glass electrode. Bruehlman and Verhoek [12] determined - $\log K_{b h}$ in a $0.5-M$ solution of potassium nitrate from data obtained with the glass electrode, and reported values of 9.74 at $25^{\circ}$ and 9.51 at $35^{\circ}$. Apparently the most precise and extensive of the earlier investigations of the dissociation of ethanolamine is that of Sivertz, Reitmeier, and Tartar [13], who determined $K_{b}$ at $25^{\circ}$ from measurements of the electrolytic conductance of aqueous solutions of ethanolamine and ethanolammonium chloride. Their result leads to a value of 9.500 for $-\log K_{b h}$, in excellent agreement with 9.498 given in table 2 .

Several factors combine to determine the effect of substituents on the strength of organic bases. Some of the most important are polar (inductive) effects, resonance, electrical repulsion, solvation, and steric effects [14 to 19]. As a result of their electrondonating or electron-attracting powers, substituent groups increase or decrease the base strength, in the Lewis sense, of the nitrogen atom of ammonia. This nitrogen atom is itself so strongly electron-attracting that each hydrogen atom of ammonia acquires a definite positive charge. Their combined repulsions make ammonia a weak base. On the other hand, the basic strength of ethylamine is 26 times as great as that of ammonia. Not only does the ethyl group have a small electron-donating effect, but substitution also reduces considerably the net repulsions of the hydrogens. The electron-attracting power of the hydroxyl group is relatively large, however, and consequently ethanolamine is only one-fourteenth as strong a base as ethylamine, or about twice as strong as ammonia.

\section{Thermodynamic Quantities}

The thermodynamic quantities for the dissociation of ethanolammonium ion (eq 2) in the standard state were computed from the constants of eq 10 by application of the usual formulas [5]. The results are summarized in table 3 . The thermodynamic functions at $25^{\circ}$ for the basic dissociation of ethanolamine in water (eq 4) are obtained by subtracting the values given in table 3 from the corresponding quanties for the ionization of water at $25^{\circ}$. The latter are listed in calories by Harned and Owen [6] and are converted to joules by multiplying each figure by 4.1840. For the basic dissociation, $\Delta F^{\circ}$ is found to be $25,675 \mathrm{j} \mathrm{mole}^{-1}, \Delta H^{\circ}$ is $6,023 \mathrm{j} \mathrm{mole}^{-1}, \Delta S^{\circ}$ is $-65.9 \mathrm{j}$ $\mathrm{deg}^{-1} \mathrm{~mole}^{-1}$, and $\Delta C_{p}^{\circ}$ is $-190 \mathrm{j} \mathrm{deg}^{-1} \mathrm{~mole}^{-1}$. These values do not differ greatly from those for the basic dissociation of ammonia [5].2

\footnotetext{
2 For ammonia, $\Delta H^{\circ}=4,345 \mathrm{j} \mathrm{mole}^{-1}, \Delta S^{\circ}=-76.4 \mathrm{j}$ : $\mathrm{deg}^{-1} \mathrm{~mole}^{-1}$, and $\Delta C_{p}^{\circ}=$ $-195 \mathrm{j} \mathrm{deg}^{-1} \mathrm{~mole}^{-1}$. The negative sign has been omitted from $\Delta C_{p}^{\bullet}$ in table 7 of [5].
}

TABLE 3. Thermodynamic quantities for the acidic dissociation of ethanolammonium ion

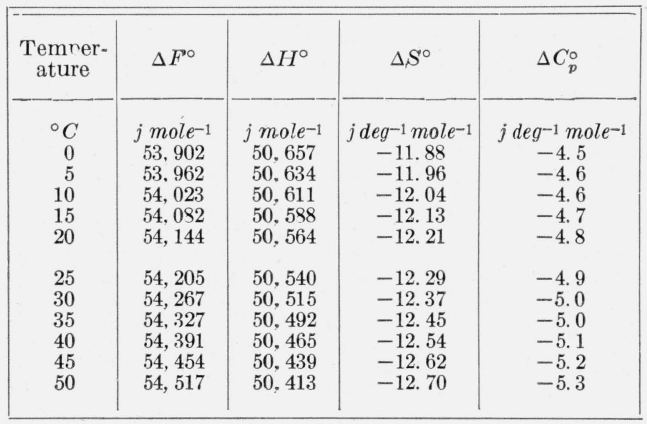

The change of heat capacity that accompanies the dissociation of ethanolammonium ion is found to be small and negative, in contrast to the rather large positive values for the methyl-substituted ammonium ions [4]. This difference can be attributed to differences in the chemical properties of the two amines. Widely different values of $\Delta C_{p}^{\circ}$ are often found for a series of neutral acids or for negatively charged acids, even though the individual dissociation reactions are of the same electric type. The close correspondence between the heat-capacity changes for the dissociation of ammonium and ethanolammonium ions may signify a reduction, by the $\mathrm{OH}$ substituent, of the hydrophobic character of the alkyl group to which Everett and Wynne-Jones ascribe the positive values found for the methyl-substituted ammonium ions.

\section{References}

[1] R. W. Gurney, J. Chem. Phys. 6, 499 (1938).

[2] E. C. Baughan, J. Chem. Phys. 7, 951 (1939).

[3] D. H. Everett and W. F. K. Wynne-Jones, Proc. Roy Soc. (London) 169A, 190 (1938).

[4] D. H. Everett and W. F. K. Wynne-Jones, Proc. Roy. Soc. (London) 17\% A, 499 (1941).

[5] R. G. Bates and G. D. Pinching, J. Research NBS 42, 419 (1949) RP1982.

[6] H. S. Harned and B. B. Owen, The physical chemistry of electrolytic solutions, 2d ed., p. 485, 514 (Reinhold Publishing Corp., New York, N. Y., 1950).

[7] F. Hückel, Physik. Z. 26, 93 (1925).

[8] G. G. Manov, R. G. Bates, W. J. Hamer, and S. F. Acree, J. Am. Chem. Soc. 65, 1765 (1943).

[9] R. G. Bates, G. D. Pinching, and E. R. Smith, J. Research NBS 45, 418 (1950) RP2153.

[10] H. S. Simms, J. Phys. Chem. 32, 1121 (1928).

[11] S. Glasstone and A. F. Schram, J. Am. Chem. Soc. 69, $1213(1947)$

[12] R. J. Bruehlman and F. H. Verhoek, J. Am. Chem. Soc. 70, 1401 (1948)

[13] V. Sivertz, R. E. Reitmeier, and H. V. Tartar, J. Am. Chem. Soc. 62, 1379 (1940).

[14] S. R. Palit, J. Indian Chem. Soc. 25, 127 (1948)

[15] H. C. Brown, H. Bartholomay, Jr., and M. D. Taylor, J. Am. Chem. Soc. 66, 435 (1944).

[16] H. C. Brown, J. Am. Chem. Soc. 67, 378 (1945).

[17] H. C. Brown, J. Am. Chem. Soc. 67, 1452 (1945).

[18] G. E. K. Branch and M. Calvin, The theory of organic chemistry, chapter VI (Prentice-Hall, Inc., New York, N. Y., 1946).

[19] A. F. Trotman-Dickenson, J, Chem. Soc., 1293 (1949).

Washington, November 28, 1950. 